\title{
Optimization methodology based on neural networks and box-behnken design applied to photocatalysis of acid red 114 dye
}

\author{
Alok Garg $^{1^{\dagger}}$, Gaganpreet Kaur ${ }^{2}$, Vikas K. Sangal ${ }^{3^{\dagger}}$, Pramod K. Bajpai ${ }^{4}$, Sushant Upadhyay ${ }^{3}$ \\ ${ }^{1}$ Department of Chemical Engineering, National Institute of Technology, Hamirpur, India \\ ${ }^{2}$ Department of Electronics and Communication Engineering, Thapar Institute of Engineering \& Technology, Patiala, India \\ ${ }^{3}$ Department of Chemical Engineering, Malaviya National Institute of Technology, Jaipur, India \\ ${ }^{4}$ Department of Chemical Engineering, Thapar Institute of Engineering \& Technology, Patiala, India
}

\begin{abstract}
The present work deals with the modeling and optimization of photocatalytic degradation $\left(\mathrm{UV} / \mathrm{TiO} \mathrm{O}_{2}\right)$ of aqueous solution of Acid Red 114 (AR114) dye using Artificial Neural Networks (ANN) and RSM. Photocatalytic treatment of $\mathrm{AR} 114$ has been executed using suspension TiO ${ }_{2}$ catalyst for commercial applications exposed to ultraviolet irradiation in a shallow pond reactor. ANN optimization has been applied to for predicting the behavior of photocatalysis. The input parameters used for analysis of aqueous dye solution are $-\mathrm{TiO}_{2}$ dose, $\mathrm{pH}$ of the dye solution, initial dye concentration, UV light intensity, time and area/volume, and time whereas the outputs are evaluated in form of degradation and decolorization efficiency of AR114. The outcomes of ANN optimization have been experimentally validated. Results achieved establish ANN modeling as a good predictive model. Parameteric optimization using multi-parameter optimization has been employed with desirability function approach. Results obtained from RSM are in line as per the results of ANN modeling as well as experimental. First order kinetics is use to effectively express degradation and decolorization of AR114 dyes. Total organic carbon (TOC) removal and GC-MS study of the dye shows the total mineralization and formation of non-toxic intermediate products.
\end{abstract}

Keywords: ANN modeling acid red 114 (AR114), Decolorization, Degradation, Photocatalysis, RSM

\section{Introduction}

Environmental safety and the improvement of environmental issues are the main concern for an actual development of life and for a sustainable development. Water is utilized in various human needs such as agriculture, industry, domestic, and energy production. Globally, domestic use represents only $15 \%$ of the total water consumption, while $25 \%$ is used in industrial activities. The major share of water constituting the remaining $60 \%$ is consumed in the agriculture activities. Associated to each of these activities, different types of pollutants are generated. Of all users, the industrial wastewater streams are posed a severe threat to human life, plants and animals and also to ecosystems of receiving water bodies. Industrial effluents from textile, pulp and paper, pharmaceutical and other chemical industries $[1,2]$ are the major source of ground and surface water pollution.

It has been predicted that approximately $15 \%$ of the dye is discharged as wastewater from dyeing industry [3]. The dye color is typically the first pollutant to be identified in the wastewater, which is produced due to the synthetic dyes in the industries. Considering both effluent compositions and volumes discharged, the wastewater produced by the textile industry is the most significant pollutant amongst the various industries. Specified the large diversity of dyes, fibers, finishing products and process aids in the application, the textile industries produce the wastewater with a large chemical diversity, quantity, and complexity. Because of increasing demands in textiles, synthetic organic dyes are extensively used in the dying process for textile fiber like polyester and cotton [4]. Besides the textile industry, the use of synthetic dyes is finding increased application in industries as petroleum,
This is an Open Access article distributed under the terms of the Creative Commons Attribution Non-Commercial License (http://creativecommons.org/licenses/by-nc/3.0/) which permits unrestricted non-commercial use, distribution, and reproduction in any medium, provided the original work is properly cited.

Copyright (C) 2020 Korean Society of Environmental Engineers
Received June 13, 2019 Accepted October 17, 2019

${ }^{\dagger}$ Corresponding author

Email: alok.garg.chem@gmail.com (Garg), vksangal.chem@mnit.ac.in Tel: +91-98765-63990

ORCID: 0000-0002-5596-0610 
leather, paper, food products and plastics [5]. The dyes are used to color the products and the residuals are discharged into the environment, particularly aquatic environment. Synthetic dyes are difficult to biodegrade because of their complex aromatic nature. Thus, the removal of dye color from wastewater is a challenging problem. The wastewater containing textile dyes are toxic and carcinogenic. Synthetic dyes such as azo dyes (having one or more azo bonds) account for more than half of the usage of dying in the textile industry and hence are the major source of pollutants in the wastewater. Unfortunately, the dyes, mostly azo dyes with aromatic structures, are recalcitrant in nature. The azo dyes are difficult to degrade by aerobic digestion and steady in oxidizing agents [6]. Additionally, wastewater from the dying processes is at a high temperature and different $\mathrm{pH}$, allowing it to hold a high amount of the color elements. These concerns have been directed to new and/or stringent rules regarding wastewater discharge from the dying industry and require more effective treatment methods.

Though problem of color removal/ COD removal finds sufficient reference in recent research, no significant work has been on the dye degradation. Photo catalytic process for water treatment is generally preceded by decolorization that is removal of the dye color at the wavelength of maximum absorption. This requires study of degradation of dye color before photocatalysis is carried out. Most of the previous work is focuses on degradation efficiency in context of color removal and/or COD removal, thereby wrongly reflecting color removal as dye degradation [7, 8]. Depending on the method used for wastewater treatment, the dye may be existing in the wastewater in its existing or in its degraded form. This demands both dye degradation and color removal be studied independently to measure the true efficiency of water treatment method used.

Most of the techniques do not reduce contaminants but they transfer pollutants from one stage to another stage. Some techniques are selective but slow to moderate in degradation rate. Other alternative methods produce more harmful intermediates and some methods require more treatment time [9]. The inability of traditional wastewater treatment techniques to effectively destroy the bio-recalcitrant contaminants led to exploring new alternatives. The advanced oxidation processes (AOPs) has appeared as one of the most efficient technologies for the degradation of the contaminants/pollutants/toxicants [10]. AOPs are further classified as homogeneous and heterogeneous processes.

In the process of heterogeneous photocatalysis, the near UV irradiation is use to the photo-excitation of a semiconductor-based photocatalyst in the existence of oxygen. Beneath the given conditions, oxidizing agents, either bound hydroxyl radicals or generate free holes. Using heterogeneous photocatalysis, organic pollutants (textile dyes) could be totally mineralized, reacting with the oxidizing agents to produce $\mathrm{CO}_{2}, \mathrm{H}_{2} \mathrm{O}$ and a low concentration of organic acids. Instead of near UV region, this process could be carried out using the close to the solar band $(\lambda<380 \mathrm{~nm})$ [1]. The semiconductor-based catalyst used in the analysis can either be in powder form suspended in the water or it may be fixed on a solid support.

Among the various semiconductors employed, the anatase phase of $\mathrm{TiO}_{2}$ (like $\mathrm{WO}_{3}, \mathrm{TiO}_{2}, \mathrm{ZnO}, \mathrm{Fe}_{2} \mathrm{O}_{3}, \mathrm{CdS}, \mathrm{ZnS}, \mathrm{V}_{2} \mathrm{O}_{5}, \mathrm{ZrO}_{2}, \mathrm{SnO}_{2}$, $\mathrm{CeO}_{2}$, and $\mathrm{Sb}_{2} \mathrm{O}_{4}$ ) is known to be a good photocatalyst for the degradation of several pollutants due to its high photosensitivity and large bandgap. The large bandgap of $\mathrm{TiO}_{2}$ of the order of 3.2 $\mathrm{eV}$ makes it difficult for charge carriers to be generated by thermal excitation. This instead requires absorption of the photon with energy $\geq 3.2 \mathrm{eV}$, which corresponds to UV light of wavelength $<380 \mathrm{~nm}$.

Parameters affecting the photocatalytic degradation of organic dyes have been studied such as initial dye concentration, photocatalyst dose, initial $\mathrm{pH}$, light irradiation and reaction time [11-14]. The photocatalysis of organic dye degradation also depends on the geometry of the reactor i.e. area to volume ratio. This parameter has not been considered so far for the modelling of photocatalysis of organic dye degradation. The complexity of the processes limits the conventional mathematical modeling techniques [15] and necessitates the use of multivariate correlation [16] to solve these complex quandaries.

Artificial neural networks (ANNs) are efficient modeling and optimization tool capable of effectively solving the nonlinear systems, especially when of more than one process parameters are involved. ANN is capable to solve the system without requiring previous information of the interactions of process variables ([17]). Kasiri et al [18] applied ANN for the degradation of Acid Red 14 dye by the photo-Fenton process and found that the initial concentration of the dye and initial $\mathrm{pH}$ to be more influential parameters in the degradation process. Khataee [19] applied ANN for the degradation of Basic Red 46 by heterogeneous photocatalysis and found that the reaction time, $\mathrm{pH}$, initial dye concentration and UV light intensity have the importance of 26.75, 27.03, 30.75 and $15.47 \%$, respectively. Khataee and Zarei [20] and Zarei et al [21] developed an ANN model with feedforward backpropagation for the photodegradation of Direct Yellow 12 and Basic Red 46 dyes using photoelectron-Fenton process and found a reasonable predictive performance. To choose the model of neural network different approaches have been evolved, most commonly being the hit and trial method. The number of the input layer and output layer neurons come from the inputs and outputs used. For hidden layer hit and trial method of a testing different number of neurons in one or two hidden layers and evaluating each topology on basis of MSE we identified an ANN model with 6:7:2:2 structures. From literature, only single work [22] could be identified with using ANN model with two hidden layers having a large number of neurons as 24 and 15, respectively in hidden layer 1 and hidden layer 2. In many cases, a large number of neurons in hidden layer may cause of over train the network. Every model has the significant contribution of input parameters by which it has developed. So far the influence of parameters for developing the model or percentage importance of parameters for model development has not been studied.

Previous studies in this field have primarily focused on traditional one factor at a time experiments. Analyzing one factor individually not only makes the process time consuming and tedious but is marred by the inability to capture the effect of interaction between different the parameters. Since photocatalytic degradation analysis requires study of more than one factor, conventional optimization techniques are insufficient to study the complex interactions involved. Response surface methodology (RSM) in systematic opti- 
mization is a suitable technique capable of addressing the complexity of more than one influencing factors. It makes possible to estimate linear interaction and quadratic effects of the input process parameters and to establish a mathematical relationship for the prediction of the responses. RSM has been used to optimize process parameters for various processes [23-26]. Chao and Zoh [27], Khataee et al [28], and Khataee et al [29], have also applied RSM methodology to model and optimize $\mathrm{UV} / \mathrm{TiO}_{2}$, photo-Fenton, and visible light $/ \mathrm{TiO}_{2}$ processes.

In the present work, both ANN coupled with RSM have been used for optimizing the photocatalysis process. The proposed study was undertaken with the objectives: (1) Degradation of Acid Red 114 by the photocatalytic process using $\mathrm{UV} / \mathrm{TiO}_{2}$ and optimizing the parameters like $\mathrm{pH}$, catalyst loading, initial concentration of dye, area to volume ratio, UV light intensity and time. (2) Modeling and optimization of the process parameters by artificial neural network and response surface methodology (3) comparison of the results obtained by ANN, RSM, and experiments (4) Reaction kinetics and total mineralization study by total organic carbon (TOC) analyzer. (5) Identification of relative contribution of the parameters for decolorization and degradation

\section{Materials and Methods}

\subsection{Photo Degradation of AR114}

The photocatalytic degradation studies of AR114 (Sigma Aldrich) were conducted using a shallow pond slurry reactor as discussed in our previous article [11]. A batch type slurry pond reactor was fabricated for performing the experiments. A Borosilicate glass vessel of $250 \mathrm{~mL}$ volume was used as the shallow pond slurry reactor and was irradiated with UV rays. The photo-reactor was fitted with $8 \mathrm{UV}$ black tubes of $36 \mathrm{~W}$ each (make of Philips Inc.), fitted in parallel on the top of the reactor. The UV lamps emit radiation in the range of $300-400 \mathrm{~nm}$, with the peak intensity at $350 \mathrm{~nm}$. This reactor is placed on a magnetic stirrer to possess the slurry in the reactor well mixed so that the $\mathrm{TiO}_{2}$ (Degussa P25) remains suspended and the concentration of the pollutant within the reactor could be assumed to be constant at any time.

The adsorption/desorption of dye on the catalyst was allowed for $30 \mathrm{~min}$ in dark condition. An aliquot of $5 \mathrm{~mL}$ was taken from the reactor with the help of a syringe. After $30 \mathrm{~min}$, UV lights were switched on. The door of the UV chamber was kept closed so that no UV rays come out. After the regular time interval of $30 \mathrm{~min}, 5 \mathrm{~mL}$ sample is withdrawn from the reactor with the help of a syringe and diluted by 1:1 ratio each time. This process of photocatalysis was allowed to continue for the next $180 \mathrm{~min}$. With an objective to filter the suspended $\mathrm{TiO}_{2}$ particles from the dye sample, centrifugation is done at the high speed of 15,000 rpm for $10 \mathrm{~min}$. While centrifugation is done it has to be ensured that the level of the sample in each centrifuge tube is same. As a result, the $\mathrm{TiO}_{2}$ particles get settled at the bottom of the tube.

A computer-based UV spectrophotometer was used for determining the concentration of samples. The decolorization and degradation efficiency were measured in terms of absorbance using spectrophotometer at wavelengths 522 and $373 \mathrm{~nm}$, respectively. The conversion percent of dye has been obtained at different intervals. The degradation and decolorization $(\mathrm{Y})$ are given by Eq. (1)

$$
Y=\frac{C_{o}-C_{t}}{C_{o}}
$$

Where $\mathrm{C}_{o}$ is the initial concentration of dye and $\mathrm{C}_{t}$ is the concentration of dye at time $t$.

Using the set-up described above degradation and decolorization of dye has been determined. A series of experiments have been conducted to achieve optimized values of all the input parameters considered- initial concentration, $\mathrm{pH}$ and catalyst loading, UV light intensity, area to volume ratio and time.

\subsection{Modeling by Artificial Neural Network}

The concept of ANN is based on massive parallelism of the human brain making it capable of establishing a relationship between a set of inputs and outputs. Of all ANN Multilayer Feedforward networks are dominant in problem-solving and optimization. It is a fully connected network which generally uses back propagation learning algorithm for the training of network. The basic architecture of the MLF network is as shown in Fig. 1(a).

It comprises an input layer of neurons representing the inputs to the problem and set of output layer neurons representing the output variables. In between the input and output are hidden layers with a variable number of neurons. Depending on the complexity of the problem number of hidden layers may vary from one to any number as required. Further, each hidden layer can have the same or a different number of neurons. Once all layers of the network are defined every neuron in one layer is connected to every neuron in the next layer using a weighted connection. This is called a fully connected network. Ns in hidden and the output layer are governed by a neuron output function and a bias function. The role of the training algorithm used is to change the weights of connections between different layers so as to achieve the desired output.
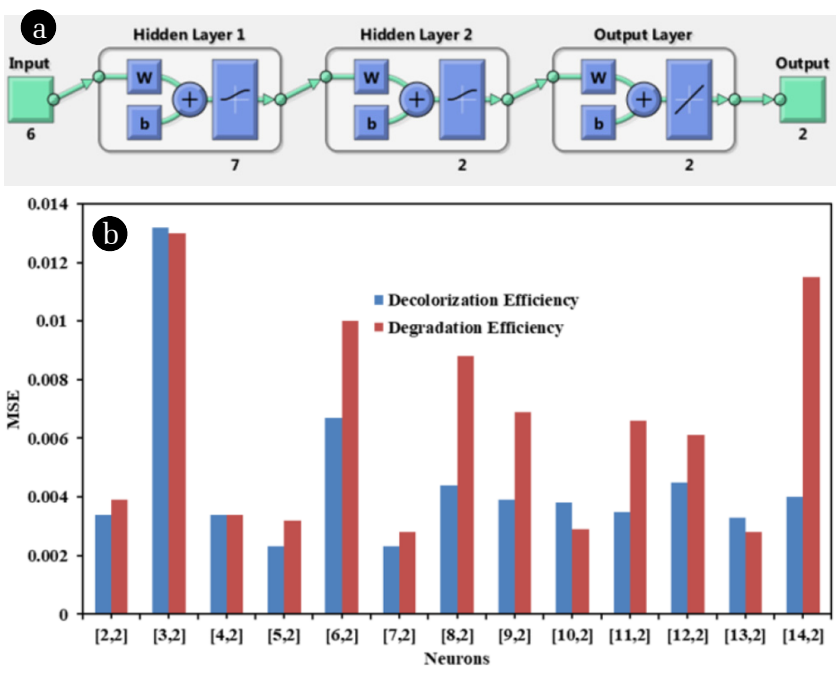

Fig. 1. (a) ANN architecture (b) Effect of the number of neurons in the hidden layer. 
In our problem, a four-layered feedforward network has been used with two hidden layers. To limit the output between 0 and 1 log-sig has been used as the output function and back-propagation learning algorithm has been used for network training.

\subsection{Multi Response Optimization Using RSM}

RSM is based on the statistical design of experiments [30] evaluating the effects of various process parameters, and identifying optimum conditions for desirable responses [31, 32]. RSM covers a number of statistical techniques including Box-Behnken design (BBD) for model building and the use of the model. BBD helps identify the interactive effects of various input process parameters on the outputs (responses). The number of experimental and/or simulation runs required to evaluate the probable inter-parametric effects on photocatalytic degradation and decolorization of dyes too are optimized using RSM.

Consider a process or system to be examined using RSM with a response $\mathrm{E}$, where $\mathrm{E}$ depends on the input parameters $\mathrm{p}_{1}, \mathrm{p}_{2}$ . . . $\mathrm{p}_{\mathrm{n}}$. The correlation between the output (response) and the input process parameters is designated by function ' $\mathrm{k}$ ' as:

$$
E=k\left(p_{1}, p_{2}, p_{3}, p_{4} \ldots \ldots \ldots p_{n}\right)
$$

Where ' $\mathrm{k}$ ' is the real response function and is unknown, $\varepsilon$ is the residual error. A second-order polynomial, eq. (3), has been used through nonlinear regression to fit the experimental/simulated data and to identify the significant model terms. Ruminating all the linear terms, linear-by-linear interaction terms, and square terms, the quadratic response model can be represented as [33]:

$$
E=m_{0}+\sum_{i=1}^{n} m_{i i} p_{i}+\sum_{i=1}^{n} m_{i i} p_{i}^{2}+\sum \sum_{i<j} m_{i i} p_{i} p_{j}+\varepsilon
$$

Where $\mathrm{m}_{0}$ is constant, $\mathrm{m}_{\mathrm{i}}$ is the slope accounting for the linear effect of the input factor $\mathrm{p}_{\mathrm{i}}, \mathrm{m}_{\mathrm{ij}}$ is the linearly mapped interaction effect between the input factors $p_{i}$ and $p_{j}$, and $m_{i i}$ is the quadratic effect of input factor $p_{i}$ [31].

These designs are preferred for the fitting of the second order models. In general, the second order model is sufficed and provides a good prediction throughout the region of interest. To choose the best model suitable to the problem under consideration generally sequential F-test accompanied by other competence measures are used [34]. The F-test is the most commonly employed method for comparing statistical models that have to fit a data set, used to recognize the model that best fits the population from which the data has been sampled. The least squares method is also a popularly used method to evaluate the parameters in approximating the polynomials. The response surface analysis is then performed using the fitted surface. If the fitted surface is a good approximation of the real response function, the analysis of the fitted surface will be approximately comparable to the analysis of the real system.

In the proposed study multi-response process optimization with desirability function approach has been applied to optimize the photocatalytic process parameters. The desirability, $d_{i}$, lies between
0 and 1 , signifying the closeness of a response to its ideal value [33]. Overall desirability $D_{e}$ is achieved by the combined effect of individual desirability functions using:

$$
D_{e}=\left(d_{1} \times \mathrm{d}_{2}\right)^{\frac{1}{2}} \text { such that, } 0 \leq \mathrm{D}_{\mathrm{e}} \leq 1
$$

Where $d_{1}$ and $d_{2}$ are the individual desirability values for the first and second response, respectively.

RSM based BBD has been used to the simulated data using the Design-Expert software version 11 (STAT-EASE Inc., Minneapolis, US). The simulated data has been analyzed by the regression analysis to fit the equations developed and also for the assessment of the statistical significance of the equations.

\section{Result and Discussion}

\subsection{Modeling and Optimization}

\subsubsection{Artificial Neural Network Model Development}

A four-layered feed-forward neural network (6:7:2:2) has been used in the proposed work for modeling of $\mathrm{Y}_{1}$ and $\mathrm{Y}_{2}$. The network is trained using a supervised learning algorithm back-propagation. The range of input and output values used in this analysis has been listed in Table 1.

ANN model gives the flexibility to vary the number of hidden layers. To choose the optimized network configuration, initially, one hidden layer was used and the number of neurons in the hidden layer was varied from 2 to 14 . Each topology was evaluated for reduction in mean square error (MSE) between expected and achieved outputs. Increasing hidden layer neurons beyond 14 resulted in an increase in MSE. Next, another hidden layer was added with 2 neurons and again each topology with neurons of first layer varying from 2 to 14 results was evaluated for MSE. The lowest value of MSE for both the outputs has been achieved for configuration of $(6: 7: 2: 2)$ as can be seen in MSE variations with hidden neurons in Fig. 1(b). Each topology was carried out twice to remove arbitrary connections if any due to random initialization of weights in the network.

In this work, the proposed network has been first trained on the set of data values of input with known output using Levenberg-Marquardt (LM) algorithm. A data set of 145 values from the experimental data was used for ANN training. To test the results a set of 23 values has been used. The results (Fig. 2) show the regression analysis for predicted results using ANN and experimental values achieved for $\mathrm{Y}_{1}$ and $\mathrm{Y}_{2}$. In Fig. 2 analysis of experimental and predicted values of $\mathrm{Y}_{1}$ and $\mathrm{Y}_{2}$ show a good approximation of output using ANN with $\mathrm{R}^{2}$ values of 0.9924 and $0.9983 \mathrm{Y}_{1}$ and $\mathrm{Y}_{2}$, respectively, which establishes the ability of proposed ANN model to effectively predict the outputs.

The weight matrix of the developed ANN model has been used to evaluate the relative contribution of the various input parameters towards the output parameters. The expected relative importance of each input parameter on the output is computed based on an equation derived from the partitioning of connection weights of ANN used: 

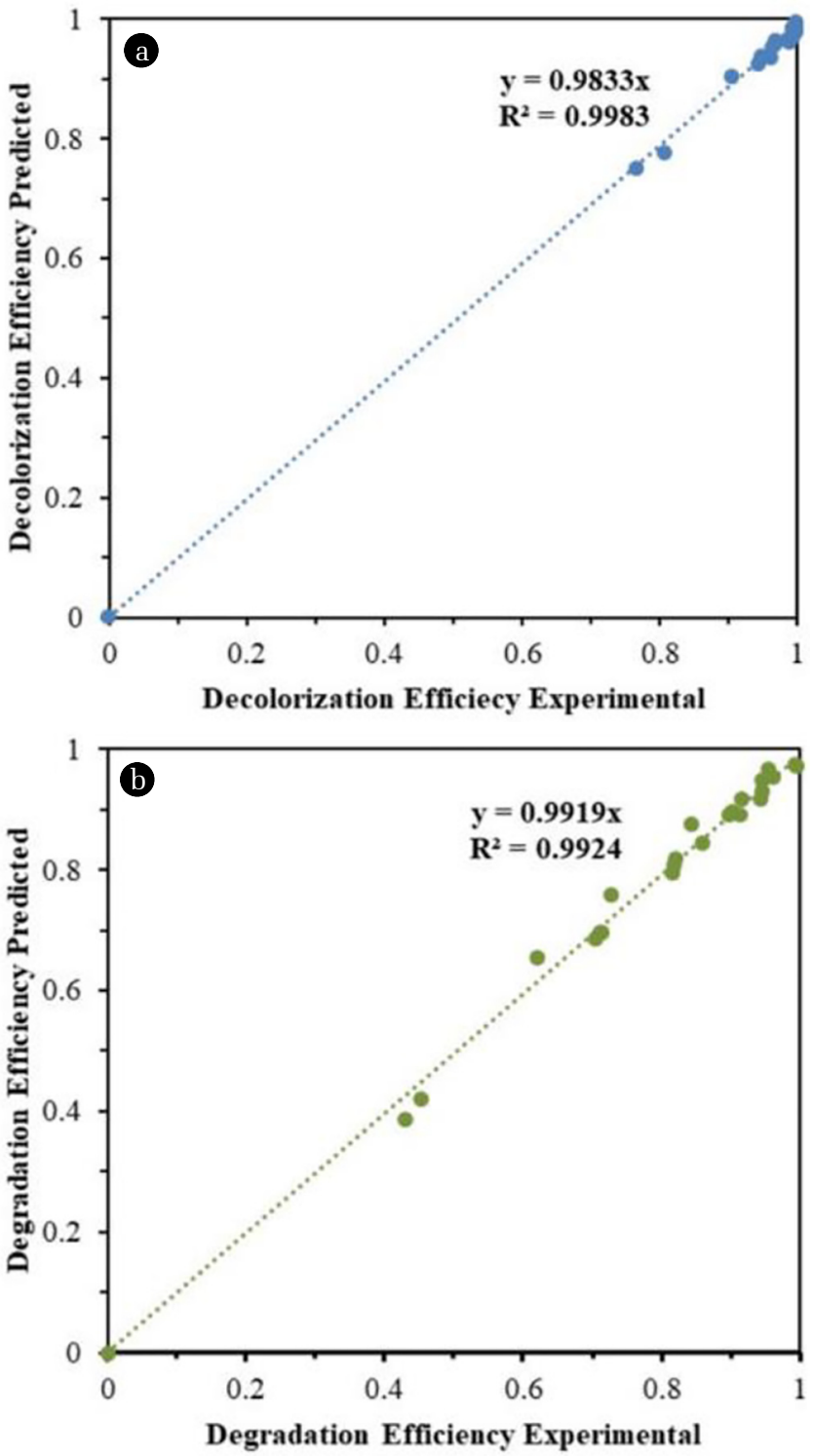

Fig. 2. Comparison of the experimental results with those predicted values via ANN modelling for the test set (a) $Y_{2}$ and (b) $Y_{1}$.

$$
\mathrm{I}_{j}=\frac{\sum_{l=1}^{N_{h 1}}\left(\left(\left|W_{j l}^{i h_{1}}\right| / \sum_{k=1}^{N_{i}}\left|W_{k l}^{i h 1}\right|\right) \sum_{m=1}^{N_{h_{2}}}\left|W_{l m}^{h_{1} h_{2}}\right|\right)\left|W_{m n}^{h_{2} o}\right|}{\sum_{k=1}^{N_{i}}\left\{\sum_{l=1}^{N_{h 1}}\left(\left(\left|W_{j l}^{i h_{1}}\right| / \sum_{k=1}^{N_{i}}\left|W_{k l}^{i h 1}\right|\right) \sum_{m=1}^{N_{h_{2}}}\left|W_{l m}^{h_{1} h_{2}}\right|\right)\left|W_{m n}^{h_{2} o}\right|\right\}}
$$

Where $I_{j}$ is the measure of the relative contribution of the $j^{\text {th }}$ input factor on the output. Here subscripts $\mathrm{k}, \mathrm{l}, \mathrm{m}$, and $\mathrm{n}$ represent the input layer, first hidden layer, the second hidden layer, and output layer neurons, respectively. $\mathrm{N}_{\mathrm{i}}$ is the number of neurons in the input layer; $\mathrm{N}_{\mathrm{h} 1}$ and $\mathrm{N}_{\mathrm{h} 2}$ are the numbers of neurons in the first and second hidden layers, respectively. The relative importance of input parameters on outputs, $\mathrm{Y}_{1}$, and $\mathrm{Y}_{2}$ as evaluated

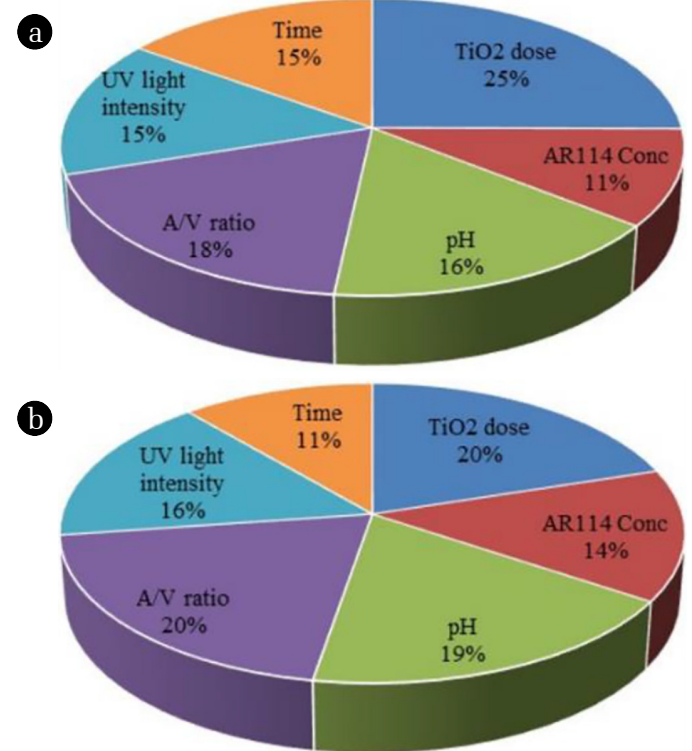

Fig. 3. Relative contribution of the parameters for (a) Decolorization and (b) Degradation efficiencies, respectively.

using Eq. (5) have been shown in Fig. 3. All parameters showed strong effects on the dye degradation and decolorization efficiencies, the $\left[\mathrm{TiO}_{2}\right]_{\mathrm{o}}$ was most effective than other parameters. $\mathrm{A} / \mathrm{V}$ is the second largest influence parameter for the degradation and decolorization of AR114.

\subsubsection{Statistical Analysis using BBD}

To validate the findings of ANN modeling three-level BBD has been applied. BBD evaluates the photocatalysis process parameters which affect the degradation and decolorization efficiency. A range of values of the input parameters- $\left[\mathrm{TiO}_{2}\right]_{\mathrm{o}},[\mathrm{AR} 114]_{0},[\mathrm{pH}]_{\mathrm{o}}, \mathrm{A} / \mathrm{V}$, $\mathrm{LI}$, and $\mathrm{t}$ has been used for the optimization.

Table 1 shows the input parameters used along with values and ranges. The 3-factor levels for BBD used can be interpreted as -1 (low), 0 (central point or middle), and 1 (high) ([35]). The simulated data has been analyzed by regression analysis to fit the equations developed and also for the assessment of the statistical significance of the equations. The input parameters used in the present work are given in Table 1 . The results of the $Y_{1}$ and $Y_{2}$ for photo-degradation of AR114 have been analyzed conferring to the design matrix as proposed by BBD.

Table 1. Results of 3 Level BBD for Chosen Variables

\begin{tabular}{lccc}
\hline \multirow{2}{*}{ Variables } & \multicolumn{3}{c}{ Levels in Box-Behnken design } \\
\cline { 2 - 4 } & Low (-1) & Medium (0) & High (+1) \\
\hline$\left[\mathrm{TiO}_{2}\right]_{\circ}$ & 1.00 & 1.625 & 2.25 \\
{$[\mathrm{AR} 114]_{\mathrm{o}}$} & 25 & 112.5 & 200 \\
{$[\mathrm{pH}]_{0}$} & 2 & 6 & 10 \\
$\mathrm{~A} / \mathrm{V}$ & 0.11 & 0.185 & 0.26 \\
$\mathrm{LI}$ & 8.70 & 10.35 & 12 \\
$\mathrm{~T}$ & 0 & 90 & 180 \\
\hline
\end{tabular}



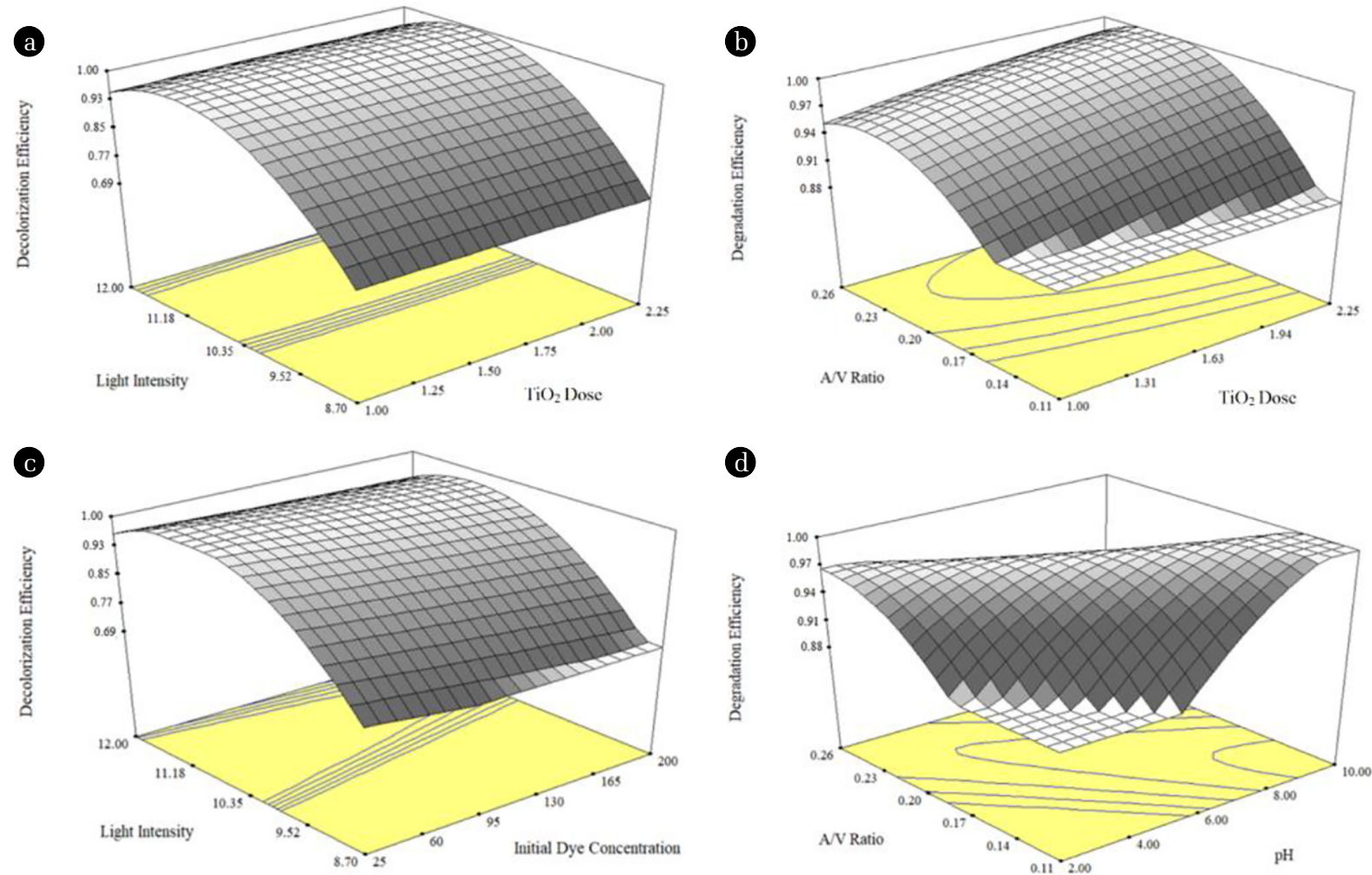

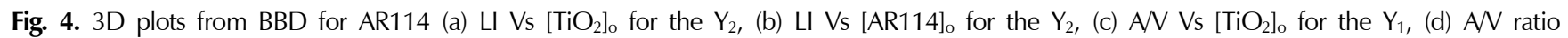
Vs $[\mathrm{pH}]_{0}$ for $\mathrm{Y}_{1}$.

As established from results above $\left[\mathrm{TiO}_{2}\right]_{\mathrm{o}}$, [AR114 $]_{\mathrm{o}}, \mathrm{LI},\left[\mathrm{TiO}_{2}\right]_{\mathrm{o}}$ * $[\mathrm{pH}]_{0},[\mathrm{pH}]_{\mathrm{o}}$ * $\mathrm{A} / \mathrm{V}$ are the highly significant parameters for degradation of AR114. On the other hand [AR114], LI, (LI) ${ }^{2},[\mathrm{AR} 114]_{\mathrm{o}}$ * $[\mathrm{pH}]_{0},[\mathrm{AR} 114]_{\mathrm{o}}{ }^{*} \mathrm{LI}$ and $[\mathrm{pH}]_{\mathrm{o}}{ }^{*} \mathrm{LI}$ are the highly significant parameters for decolorization of AR114. Findings of ANOVA are fall in line with the results from previous analysis by 37[] which reported that $\mathrm{pH}$ and initial dye concentration are the most significant process parameters for the photocatalytic degradation of methylene blue.

A manual regression method has been used to fit the second order polynomial eq. (3) to the simulated data and to recognize the significant model terms. The model summary statistic shows the regression coefficient $\left(\mathrm{R}^{2}\right)$ to be the maximum for the quadratic model for the $Y_{1}\left(R^{2}=0.9311\right)$ and $Y_{2}\left(R^{2}=0.9872\right)$. For the $Y_{1}$ and $Y_{2}$ adequate precision ratios of 11.048 and 26.503, respectively, have been obtained. For a model to efficiently a problem precision ratio of greater than 4 is required. With precision ratio higher than 4 a model effectively explore the design space [36]. The findings of the analysis of variance (ANOVA) for the $\mathrm{Y}_{1}$ and $\mathrm{Y}_{2}$ have been tabulated in Table 2. The F-value of 13.02 and $74.37 \mathrm{Y}_{1}$ and $\mathrm{Y}_{2}$, respectively establish the significance of the used model. Removal of insignificant terms from ANOVA, reduced quadratic model is obtained which summarizes the outcomes for each response and shows the significant model terms.

As observed from results data points on normal probability plot and a dot diagram of these residuals lie sufficiently nearby to the straight line which justifies the assumptions used in the investigation. Therefore, the models developed are sufficient and the minimum residuals have been obtained from the prediction of each response. The 3D response surface plots of two independent variables, while keeping the other constant, adequately give information on the individual and interaction effects of the independent variables on the responses. Fig. 5(a) and (b) show the 3D response surface plots for photocatalytic decolorization and degradation of AR114, respectively. From Fig. 4(a) and (b), it is clear that the maximum decolorization has been in the light intensity range of 11.18 to $12.00 \mathrm{Wm}^{-2}$. Results also indicate that the degradation of AR114 increases with an increase in A/V of the reactor (Fig. 4(c) and (d)) i.e. from 0.20 to $0.26 \mathrm{~cm}^{-1}$.

The accuracy of the developed models has been validated by carrying out the confirmatory experimental runs under the given test conditions. The simulation ranges used are as defined earlier. The optimized process parameters at an initial dye concentration of $80 \mathrm{mg} \mathrm{L}^{-1}$ are found to be $\left[\mathrm{TiO}_{2}\right]_{\mathrm{o}}=1.2 \mathrm{~g} \mathrm{~L}^{-1},[\mathrm{pH}]_{\mathrm{o}}=3.6$, $\mathrm{A} / \mathrm{V}=0.25 \mathrm{~cm}^{-1}, \mathrm{LI}=10.22 \mathrm{Wm}^{-2}$ and $\mathrm{T}=150 \mathrm{~min}$.

Comparative analysis of degradation and decolorization efficiency attained by BBD was found to be 1 each, from ANN model, these were 0.99 and 0.985 , respectively and from experimental runs 0.988 and 0.968 , respectively, using optimum process parameters have been tabulated in Table S1. Results in Table S1 clearly show that the optimization of process parameters for the photocatalytic decolorization and degradation of AR114 can be carried out accurately and satisfactorily using the said method. Using this 
Table 2. Results of ANOVA Model for $Y_{1}$ and $Y_{2}$

\begin{tabular}{|c|c|c|c|c|c|c|c|c|c|c|}
\hline \multirow{2}{*}{ Source } & \multicolumn{5}{|c|}{ Degradation efficiency } & \multicolumn{5}{|c|}{ Decolorization efficiency } \\
\hline & Sum of Squares & DF & Mean Square & F Value & Prob $>$ F & Sum of Squares & DF & Mean Square & F Value & Prob $>$ F \\
\hline Model & 2.025 & 27 & 0.075 & 13.02 & $<0.0001$ & 1.180 & 27 & 0.044 & 74.37 & $<0.0001$ \\
\hline$\left[\mathrm{TiO}_{2}\right]_{0}$ & 0.053 & 1 & 0.053 & 9.15 & 0.0055 & 0.000 & 1 & 0.000 & 0.81 & 0.3764 \\
\hline$[\mathrm{AR} 114]_{\mathrm{o}}$ & 0.065 & 1 & 0.065 & 11.28 & 0.0024 & 0.009 & 1 & 0.009 & 15.76 & 0.0005 \\
\hline$[\mathrm{pH}]_{\mathrm{o}}$ & 0.025 & 1 & 0.025 & 4.38 & 0.0464 & 0.002 & 1 & 0.002 & 2.76 & 0.1089 \\
\hline $\mathrm{A} / \mathrm{V}$ & 0.003 & 1 & 0.003 & 0.47 & 0.4997 & 0.001 & 1 & 0.001 & 2.09 & 0.1600 \\
\hline $\mathrm{LI}$ & 1.125 & 1 & 1.125 & 195.36 & $<0.0001$ & 0.769 & 1 & 0.769 & 1308.8 & $<0.0001$ \\
\hline $\mathrm{T}$ & 0.012 & 1 & 0.012 & 2.17 & 0.1530 & 0.001 & 1 & 0.001 & 2.06 & 0.1628 \\
\hline$\left(\left[\mathrm{TiO}_{2}\right]_{\mathrm{o}}\right)^{2}$ & 0.000 & 1 & 0.000 & 0.05 & 0.8214 & 0.000 & 1 & 0.000 & 0.14 & 0.7130 \\
\hline$\left([\mathrm{AR} 114]_{\mathrm{o}}\right)^{2}$ & 0.000 & 1 & 0.000 & 0.01 & 0.9148 & 0.000 & 1 & 0.000 & 0.13 & 0.7189 \\
\hline$\left([\mathrm{pH}]_{\mathrm{o}}\right)^{2}$ & 0.064 & 1 & 0.064 & 11.13 & 0.0026 & 0.000 & 1 & 0.000 & 0.28 & 0.6001 \\
\hline$(\mathrm{A} / \mathrm{V})^{2}$ & 0.083 & 1 & 0.083 & 14.39 & 0.0008 & 0.002 & 1 & 0.002 & 3.96 & 0.0573 \\
\hline$(\mathrm{LI})^{2}$ & 0.209 & 1 & 0.209 & 36.20 & $<0.0001$ & 0.306 & 1 & 0.306 & 521.63 & $<0.0001$ \\
\hline$(\text { Time })^{2}$ & 0.047 & 1 & 0.047 & 8.13 & 0.0084 & 0.001 & 1 & 0.001 & 1.43 & 0.2419 \\
\hline$\left(\left[\mathrm{TiO}_{2}\right] *[\mathrm{AR} 114]_{\mathrm{o}}\right.$ & 0.003 & 1 & 0.003 & 0.58 & 0.4539 & 0.000 & 1 & 0.000 & 0.32 & 0.5737 \\
\hline$\left(\left[\mathrm{TiO}_{2}\right] *[\mathrm{pH}]_{\mathrm{o}}\right)$ & 0.113 & 1 & 0.113 & 19.61 & 0.0002 & 0.000 & 1 & 0.000 & 0.45 & 0.5092 \\
\hline$\left(\left[\mathrm{TiO}_{2}\right] * \mathrm{~A} / \mathrm{V}\right)$ & 0.002 & 1 & 0.002 & 0.32 & 0.5739 & 0.000 & 1 & 0.000 & 0.02 & 0.9034 \\
\hline$\left(\left[\mathrm{TiO}_{2}\right] * \mathrm{LI}\right)$ & 0.000 & 1 & 0.000 & 0.00 & 0.9969 & 0.000 & 1 & 0.000 & 0.07 & 0.7897 \\
\hline$\left(\left[\mathrm{TiO}_{2}\right] * \mathrm{~T}\right)$ & 0.000 & 1 & 0.000 & 0.00 & 0.9974 & 0.000 & 1 & 0.000 & 0.08 & 0.7863 \\
\hline$\left([\mathrm{AR} 114]_{\mathrm{o}} *{ }^{*}[\mathrm{pH}]_{\mathrm{o}}\right)$ & 0.009 & 1 & 0.009 & 1.58 & 0.2196 & 0.006 & 1 & 0.006 & 10.29 & 0.0035 \\
\hline$\left([\mathrm{AR} 114]_{\mathrm{o}} * \mathrm{~A} / \mathrm{V}\right)$ & 0.001 & 1 & 0.001 & 0.12 & 0.7329 & 0.000 & 1 & 0.000 & 0.58 & 0.4524 \\
\hline$\left([\mathrm{AR} 114]_{\circ} * \mathrm{LI}\right)$ & 0.009 & 1 & 0.009 & 1.64 & 0.2113 & 0.006 & 1 & 0.006 & 9.94 & 0.0041 \\
\hline$\left([\mathrm{AR} 114]_{\mathrm{o}} * \mathrm{~T}\right)$ & 0.006 & 1 & 0.006 & 1.10 & 0.3049 & 0.001 & 1 & 0.001 & 1.90 & 0.1802 \\
\hline$\left([\mathrm{pH}]_{\mathrm{o}}\right.$ * A/V) & 0.256 & 1 & 0.256 & 44.38 & $<0.0001$ & 0.000 & 1 & 0.000 & 0.64 & 0.4325 \\
\hline$\left([\mathrm{pH}]_{\mathrm{o}} * \mathrm{LI}\right)$ & 0.009 & 1 & 0.009 & 1.58 & 0.2197 & 0.006 & 1 & 0.006 & 9.96 & 0.0040 \\
\hline$\left([\mathrm{pH}]_{\mathrm{o}} * \mathrm{~T}\right)$ & 0.001 & 1 & 0.001 & 0.17 & 0.6863 & 0.000 & 1 & 0.000 & 0.05 & 0.8316 \\
\hline$(\mathrm{A} / \mathrm{V} * \mathrm{LI})$ & 0.000 & 1 & 0.000 & 0.00 & 0.9968 & 0.000 & 1 & 0.000 & 0.05 & 0.8194 \\
\hline$(\mathrm{A} / \mathrm{V} * \mathrm{~T})$ & 0.000 & 1 & 0.000 & 0.03 & 0.8654 & 0.000 & 1 & 0.000 & 0.05 & 0.8284 \\
\hline$(\mathrm{LI} * \mathrm{~T})$ & 0.006 & 1 & 0.006 & 1.10 & 0.3049 & 0.001 & 1 & 0.001 & 1.86 & 0.1839 \\
\hline Residual & 0.150 & 26 & 0.006 & & & 0.015 & 26 & 0.001 & & \\
\hline Lack of fit & 0.150 & 21 & 0.007 & & & 0.015 & 21 & 0.001 & & \\
\hline Pure error & 0.000 & 5 & 0.000 & & & 0.000 & 5 & 0.000 & & \\
\hline Cor total & 2.175 & 53 & & & & 1.195 & 53 & & & \\
\hline
\end{tabular}

method fewer simulation runs are required in comparison to other techniques reported in the literature.

3.1.3. Reaction kinetics of degradation and decolorization of AR114 The reaction kinetics of degradation and decolorization of AR114 were studied using optimized conditions as shown in Table S1. Results obtained from the UV light irradiation and fitting the first order reaction kinetics ( $\ln \mathrm{C}_{\mathrm{o}} / \mathrm{C}=\mathrm{k}^{\prime} \mathrm{t}$, where, $\mathrm{C}_{\mathrm{o}}$ is the initial dye concentration, $\mathrm{C}$ is the concentration at any given time $\mathrm{t}$ ) has been plotted in Fig. 5(a). The reaction rates were found to be 0.253 and $0.0542 \mathrm{~min}^{-1}$ for $\mathrm{Y}_{1}$ and $\mathrm{Y}_{2}$, respectively.

\subsubsection{TOC Removal versus degradation and decolorization}

Total Organic Carbon (TOC) is highly significant in terms of identifying the pollutants. TOC is a very superficial, non -vague measurement of all organics contaminates present in a wastewater. Also, low amount of TOC could ensure the lack of possibly toxic organic contaminants in wastewater. In continuation of this, Fig. 5(b) shows the TOC removal versus photo-decolorization and degradation of AR114 on optimized conditions as shown in Table S1. Results show that the Photo-degradation and decolorization of AR114 continuously increasing with respect to time and it is totally decolorized 

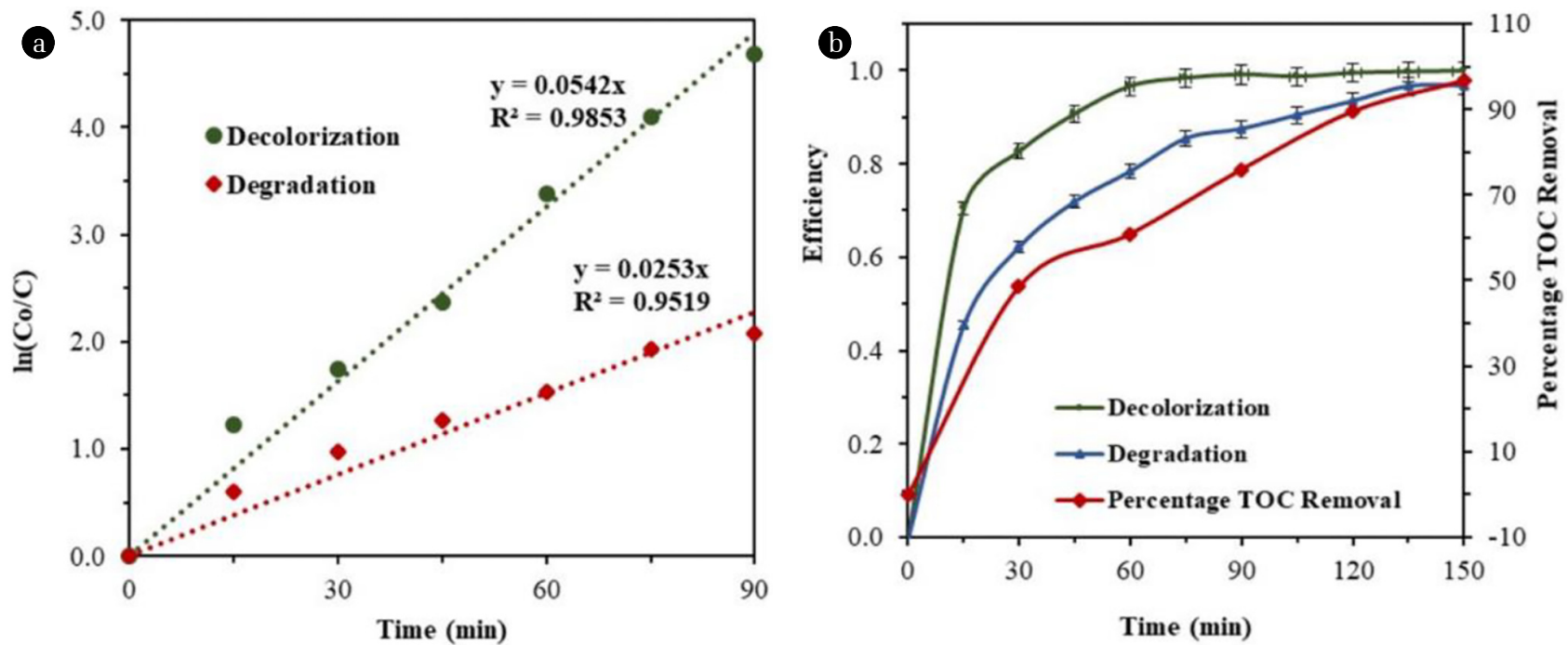

Fig. 5. (a) Reaction kinetics of AR114 (b) Dye degradation, decolorization, and TOC removal (Reaction conditions: $[\mathrm{Dye}]_{\mathrm{o}}=80 \mathrm{mg} \mathrm{L}^{-1},\left[\mathrm{TiO}{ }_{2}\right]_{\mathrm{o}}$ $=1.2 \mathrm{~g} \mathrm{~L}^{-1},[\mathrm{pH}]_{\mathrm{o}}=3.6, \mathrm{~A} / \mathrm{V}=0.25 \mathrm{~cm}^{-1}, \mathrm{LI}=10.22 \mathrm{Wm}^{-2}$ )

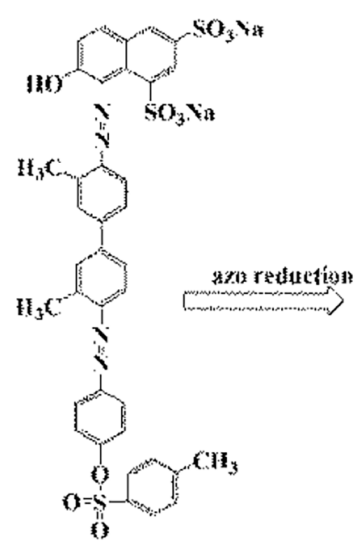

Arid $13 \mathrm{ed} 114$

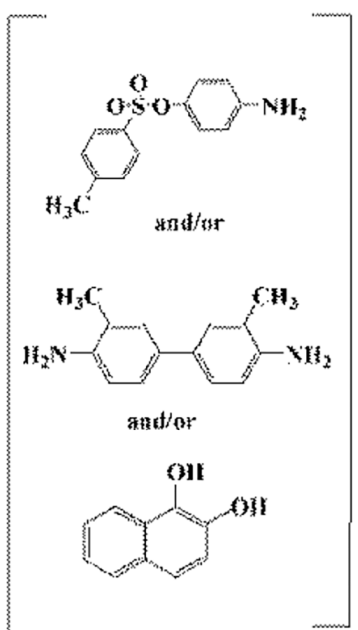

Fig. 6. Possible reaction pathways of the photodegradation of AR114.

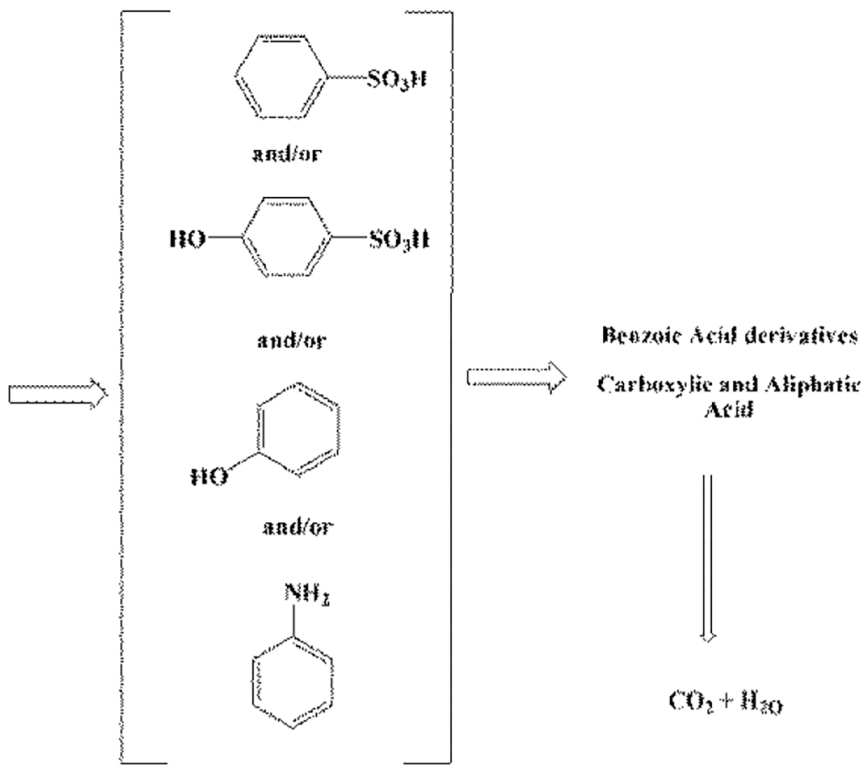

and degraded after 150 min of UV irradiation. But in the case of TOC removal, it gets slow after $30 \mathrm{~min}$ of UV light irradiation. But after 150 min UV light irradiation, TOC removal was also observed $98 \%$. This will be due to the conversion of intermediate products; later these products have also been degraded.

\subsubsection{Determination of dye degradation products/intermediates} in $\mathrm{UV} / \mathrm{TiO}_{2}$ process

The intermediate products formed during the photo-degradation of AR114 under UV light irradiation at optimized conditions as shown in Table 2 were examined by GC/MS. It has been tried to recognize the major aromatic compounds resultant from AR114 degradation. The identified intermediate products are shown in Table
S2. As it has been shown in Table S2, seven compounds were effectively noticed. It should be noted that some more chromatographic peaks have also been found but could not be completely recognized.

A possible photo-degradation reaction pathway for Acid Red 114 based on GC/MS study has been proposed as shown in Fig. 6. The AR114 dye (poly-aromatic) experienced an asymmetric cleavage by oxidation in presence of hydroxyl radical to form aromatic compounds, aniline derivative, and sulphur based aromatic compounds and intermediates. Further, as the aromatic rings of intermediate compounds break into different cleavage compounds followed by organic acids like carboxylic and aliphatic acids. These non-toxic acids further oxidized in presence of hydroxyl radical and produce carbon dioxide and water. 


\section{Conclusions}

The present study shows the efficacy of photocatalytic degradation and decolorization of AR114 employing the $\mathrm{UV}^{-} \mathrm{TiO}_{2}$. Modeling and optimization results using ANN and RSM are presented for the photocatalytic treatment of AR114. Multi-response optimization by desirability function approach was used to optimize the photocatalytic process variables.

The MSE is found minimum with seven and two neurons for first and second hidden layer, respectively for the decolorization and degradation of AR114 using the Levenberg-Marquardt (LM) algorithm with four-layered learning algorithm back-propagation in ANN network. The relative importance of the input parameters on decolorization and degradation efficiencies show the $\mathrm{TiO}_{2}$ dose as the most effective input parameter in comparison to other input operating parameters followed by area to volume ratio. For the photocatalytic treatment of AR114 dye, the optimum conditions were evaluated. The predicted values for Model $\mathrm{F}$ using ANOVA are 74.37 and 13.02 for decolorization and degradation efficiency of AR114, respectively implying that the developed model is significant. The first order kinetics was obtained for decolorization and degradation of AR114. The first order rate constants were found to be $0.0547 \mathrm{~min}^{-1}$ and $0.0253 \mathrm{~min}^{-1}$ for the decolorization and degradation, respectively of AR114. The mineralization of the dye has also been confirmed by removal of TOC as well as GC-MS analysis under the optimum conditions.

\section{Nomenctalure}

$\begin{array}{lll}\mathrm{TiO}_{2} \text { Amount g } & \mathrm{L}^{-1} & {\left[\mathrm{TiO}_{2}\right]_{\circ}} \\ \begin{array}{l}\text { Initial Dye Conc } \\ \mathrm{pH}[\mathrm{pH}]_{\mathrm{o}}\end{array} & \mathrm{mg} \mathrm{L}^{-1} & {[\mathrm{AR} 114]_{\mathrm{o}}} \\ \begin{array}{l}\text { Area to Volume (A/V) Ratio } \\ \text { Light Intensity }\end{array} & \mathrm{cm}^{-1} & \mathrm{~A} / \mathrm{V} \\ \text { Time } & \mathrm{Wm}^{-2} & \mathrm{LI} \\ \text { Degradation Efficiency } & \mathrm{min} & \mathrm{T} \\ \text { Decolorization Efficiency } & & \mathrm{Y}_{1} \\ & & \mathrm{Y}_{2}\end{array}$

\section{Author Contributions}

A.G. (Assistant Professor) conducted all the experiments and wrote the manuscript. G.K. (Assistant Professor) conducted the coding for Artificial Neural Network and wrote the manuscript. V.K.S. (Associate Professor) supervised all experiments closely and made corrections in the manuscript. P.K.B. (Professor) supervised all experiments closely and made corrections in the manuscript. S.U. (Associate Professor) conducted the coding for Artificial Neural Network and wrote the manuscript.

\section{References}

1. Malato S, Blanco J, Vidal A, Richter C. Photocatalysis with solar energy at a pilot-plant scale: An overview. Appl. Catal.
$B$ Environ. 2002;37:1-15.

2. Das S, Srivastava VC. Recent advances in fabrication of photocatalytic micro-reactor. Mater. Sci. Forum 2016;855:156-167.

3. Yadav A, Mukherji S, Garg A. Removal of chemical oxygen demand and color from simulated textile wastewater using a combination of chemical/physicochemical processes. Ind. Eng. Chem. Res. 2013;52:10063-10071.

4. Mane VS, Mall ID, Srivastava VC. Use of bagasse fly ash as an adsorbent for the removal of brilliant green dye from aqueous solution. Dyes Pigm. 2007;73:269-278.

5. Aye T, Mehrvar M, Anderson WA. Effects of photocatalysis on the biodegradability of Cibacron Brilliant Yellow 3G-P (Reactive Yellow 2). J. Environ. Sci. Health Part A 2004;39: 113-126.

6. Chen C-Y, Chang J-C, Chen A-H. Competitive biosorption of azo dyes from aqueous solution on the templated crosslinked-chitosan nanoparticles. J. Hazard. Mater. 2011;185: 430-441.

7. Hama Aziz KH. Application of different advanced oxidation processes for the removal of chloroacetic acids using a planar falling film reactor. Chemosphere 2019;228:377-383.

8. Hama Aziz KH, Mahyar A, Miessner H, et al. Application of a planar falling film reactor for decomposition and mineralization of methylene blue in the aqueous media via ozonation, Fenton, photocatalysis and non-thermal plasma: A comparative study. Proc. Saf. Environ. Protect. 2018;113:319-329.

9. Sharma J, Mishra IM, Kumar V. Mechanistic study of photo-oxidation of bisphenol-A (BPA) with hydrogen peroxide $\left(\mathrm{H}_{2} \mathrm{O}_{2}\right)$ and sodium persulfate (SPS). J. Environ. Manage. 2016;166: 12-22.

10. Sharma J, Mishra IM, Kumar V. Degradation and mineralization of bisphenol A (BPA) in aqueous solution using advanced oxidation processes: $\mathrm{UV} / \mathrm{H}_{2} \mathrm{O}_{2}$ and $\mathrm{UV} / \mathrm{S}_{2} \mathrm{O}_{8}^{-}$oxidation systems. $J$. Environ. Manage. 2015;156:266-275.

11. Garg A, Sangal VK, Bajpai PK. Decolorization and degradation of Reactive Black 5 dye by photocatalysis: Modeling, optimization and kinetic study. Desal. Water Treat. 2016;57: 18003-18015.

12. Chatterjee D, Patnam VR, Sikdar A, Joshi P, Misra R, Rao NN. Kinetics of the decoloration of reactive dyes over visible light-irradiated $\mathrm{TiO}_{2}$ semiconductor photocatalyst. J. Hazard. Mater. 2008;156:435-441.

13. Kansal SK, Kaur N, Singh S. Photocatalytic degradation of two commercial reactive dyes in aqueous phase using nanophotocatalysts. Nanoscale Res. Lett. 2009;4:709-716.

14. Mahadwad OK, Parikh PA, Jasra RV, Patil C. Photocatalytic degradation of reactive black-5 dye using $\mathrm{TiO}_{2}$ impregnated ZSM-5. Bull. Mater. Sci. 2011;34:551-556.

15. Soleymani AR, Saien J, Bayat H. Artificial neural networks developed for prediction of dye decolorization efficiency with $\mathrm{UV} / \mathrm{K}_{2} \mathrm{~S}_{2} \mathrm{O}_{8}$ process. Chem. Eng. J. 2011;170:29-35.

16. Aleboyeh A, Kasiri MB, Olya ME, Aleboyeh H. Prediction of azo dye decolorization by $\mathrm{UV} / \mathrm{H}_{2} \mathrm{O}_{2}$ using artificial neural networks. Dyes Pigm. 2008;77:288-294.

17. Dutta S, Parsons SA, Bhattacharjee C, Bandhyopadhyay S, Datta S. Development of an artificial neural network model for adsorption and photocatalysis of reactive dye on $\mathrm{TiO}_{2}$ surface. Expert 
Syst. Appl. 2010;37:8634-8638.

18. Kasiri MB, Aleboyeh $\mathrm{H}$, Aleboyeh A. Modeling and optimization of heterogeneous photo-fenton process with response surface methodology and artificial neural networks. Environ. Sci. Technol. 2008;42:7970-7975.

19. Khataee AR. Photocatalytic removal of C.I. Basic Red 46 on immobilized $\mathrm{TiO}_{2}$ nanoparticles: Artificial neural network modelling. Environ. Technol. 2009;30:1155-1168.

20. Khataee AR, Zarei M. Photocatalysis of a dye solution using immobilized $\mathrm{ZnO}$ nanoparticles combined with photoelectrochemical process. Desalination 2011;273:453-460.

21. Zarei M, Khataee AR, Ordikhani-Seyedlar R, Fathinia M. Photoelectro-Fenton combined with photocatalytic process for degradation of an azo dye using supported $\mathrm{TiO}_{2}$ nanoparticles and carbon nanotube cathode: Neural network modeling. Electrochim. Acta 2010;55:7259-7265.

22. Piuleac CG, Curteanu S, Rodrigo MA, Sáez C, Fernández FJ. Optimization methodology based on neural networks and genetic algorithms applied to electro-coagulation processes. Cent. Eur. J. Chem. 2013;11:1213-1224.

23. Kalil SJ, Maugeri F, Rodrigues MI. Response surface analysis and simulation as a tool for bioprocess design and optimization. Process Biochem. 2000;35:539-550.

24. Mannan S, Fakhru'l-Razi A, Alam MZ. Optimization of process parameters for the bioconversion of activated sludge by Penicillium corylophilum, using response surface methodology. J. Environ. Sci. 2007;19:23-28.

25. Kumar A, Prasad B, Mishra IM. Process parametric study for ethene carboxylic acid removal onto powder activated carbon using Box-Behnken design. Chem. Eng. Technol. 2007;30: 932-937.

26. Agnihotri S, Kaur P, Sangal VK, Garg A, Verma A. Parametric study for the treatment of simulated cetirizine wastewater using electrochemical methods: Optimization and cost analysis. J. Electrochem. Soc. 2018;165:E556-E562.

27. Cho I-H, Zoh K-D. Photocatalytic degradation of azo dye
(Reactive Red 120) in $\mathrm{TiO}_{2} / \mathrm{UV}$ system: Optimization and modeling using a response surface methodology (RSM) based on the central composite design. Dyes Pigm. 2007;75:533-543.

28. Khataee AR, Zarei M, Moradkhannejhad L. Application of response surface methodology for optimization of azo dye removal by oxalate catalyzed photoelectro-Fenton process using carbon nanotube-PTFE cathode. Desalination 2010;258:112-119.

29. Khataee AR, Zarei M, Moradkhannejhad L, Nourie S, Vahid B. Nitrogen doping of commercial $\mathrm{TiO}_{2}$ nanoparticles for enhanced photocatalytic degradation of dye under visible light: Central composite design approach. Adv. Chem. Lett. 2013;1:24-31.

30. Vaez M, Zarringhalam Moghaddam A, Alijani S. Optimization and modeling of photocatalytic degradation of azo dye using a response surface methodology(RSM) based on the central composite design with immobilized titania nanoparticles. Ind. Eng. Chem. Res. 2012;51:4199-4207.

31. Box GEP, Hunter JS. Multi-factor experimental designs for exploring response surfaces. Annal. Math. Stat. 1957;28:195-241.

32. Box GEP, Behnken DW. Some new three level designs for the study of quantitative variables. Technometrics 1960;2:455-475.

33. Sangal VK, Kumar V, Mishra IM. Process parametric optimization of a divided wall distillation column. Chem. Eng. Commun. 2014;201:72-87.

34. Muthukumar M, Mohan D, Rajendran M. Optimization of mix proportions of mineral aggregates using Box Behnken design of experiments. Cem. Concr. Comp. 2003;25:751-758.

35. Evans M. Optimization of manufacturing processes: A response surface approach. London: Maney Materials Science; 2003.

36. Sangal VK, Kumar V, Mishra IM. Optimization of structural and operational variables for the energy efficiency of a divided wall distillation column. Comput. Chem. Eng. 2012;40:33-40.

37. Sahoo C, Gupta AK. Optimization of photocatalytic degradation of methyl blue using silver ion doped titanium dioxide by combination of experimental design and response surface approach. J. Hazard. Mater. 2012;215-216:302-310. 